\title{
ARTICLE
}

Clinical Study

\section{Impact of primary tumour location on efficacy of bevacizumab plus chemotherapy in metastatic colorectal cancer}

\author{
Fotios Loupakis ${ }^{1}$, Herbert I. Hurwitz ${ }^{2,8}$, Leonard Saltz ${ }^{3}$, Dirk Arnold ${ }^{4}$, Axel Grothey ${ }^{5}$, Quynh Lan Nguyen ${ }^{6}$, Stuart Osborne ${ }^{6}$, \\ Jonathan Talbot ${ }^{6}$, Stefanie Srock ${ }^{6}$ and Heinz-Josef Lenz ${ }^{7}$
}

BACKGROUND: Two first-line (1L) bevacizumab trials showed the prognostic value of primary tumour location in metastatic colorectal cancer (mCRC). In this retrospective subgroup analysis, further analysis of the predictive effect of bevacizumab is presented.

METHODS: Patients with sidedness information from two randomised phase III studies of bevacizumab + chemotherapy (CT) vs CT as $1 \mathrm{~L}$ mCRC treatment were analysed retrospectively.

RESULTS: Sidedness was determined in 1590 (27\% right and 73\% left) of 2214 patients. Progression-free survival was improved with bevacizumab $+\mathrm{CT}$ vs $\mathrm{CT}$ in right-sided $(\mathrm{HR}=0.75 ; 95 \% \mathrm{Cl} 0.61,0.93 ; p=0.008)$ and left-sided $(\mathrm{HR}=0.76 ; 95 \% \mathrm{Cl} 0.67,0.86 ; p<$ 0.001 ) mCRC (pooled analysis). Similarly, overall survival was numerically improved with bevacizumab $+C T$ vs CT in right-sided $\mathrm{mCRC}(\mathrm{HR}=0.82 ; 95 \% \mathrm{Cl} 0.65,1.03 ; p=0.085)$, and significantly improved in left-sided $\mathrm{mCRC}(\mathrm{HR}=0.85 ; 95 \% \mathrm{Cl} 0.74,0.98 ; p=$ 0.028).

CONCLUSIONS: This analysis indicates that the effect of bevacizumab is independent of tumour location in mCRC.

British Journal of Cancer (2018) 119:1451-1455; https://doi.org/10.1038/s41416-018-0304-6

\section{BACKGROUND}

Metastatic colorectal cancer ( $\mathrm{mCRC}$ ) is a heterogeneous disease increasingly characterised by chromosomal and molecular variations. ${ }^{1}$ CRCs arising from the right or left side of the colon have markedly different clinical characteristics, incidence rates, and gene expression profiles. ${ }^{2-5}$ Right-sided tumours are more likely to be characterised by BRAF mutations and high microsatellite instability, whereas left-sided tumours are commonly associated with chromosomal instability and overexpression of epidermal growth factor receptor ligands. ${ }^{3}$ Right- and left-sided tumours also differ with regard to their microbiomes and host-related factors. Mucosal microbiota organisation has been shown to be a distinct feature of right-sided tumours, ${ }^{6}$ and right-sided CRC is associated with older age and fewer surgeries for urgent indications. ${ }^{7}$

Recent reports have focused on differences in prognosis and therapeutic outcome between tumours originating in the right and left side of the colon. ${ }^{1,8,9}$ In a report of two randomised phase III mCRC trials with bevacizumab, overall survival (OS) was significantly longer in left-sided vs right-sided tumours (AVF2017g: hazard ratio $(\mathrm{HR})=0.55,95 \%$ confidence interval $(\mathrm{Cl})=0.43-0.70$; N016966: $\mathrm{HR}=0.71,95 \% \mathrm{Cl}=0.62-0.82)$; these differences remained statistically significant within treatment subgroups (i.e., bevacizumab + chemotherapy (CT) or CT alone). ${ }^{8}$
Given the fundamental differences in colon tumour sidedness, further understanding of the influence of sidedness from pivotal mCRC studies may add value to our current knowledge of optimal mCRC treatments. Here we present data on the benefit of adding bevacizumab to $C T$ in patients with right- or left-sided tumours from the NO16966 and AVF2107g trials.

\section{MATERIALS AND METHODS}

Patient populations, treatment, and outcomes have been described in the AVF2107g and N016966 studies. $^{10,11}$ Briefly, AVF2107g was a randomised, placebo-controlled trial of bevacizumab with irinotecan, bolus fluorouracil, and leucovorin in patients with previously untreated mCRC. ${ }^{10}$ NO16966 was a randomised, noninferiority comparison of 5-fluorouracil, folinic acid, and oxaliplatin (FOLFOX4) vs capecitabine plus oxaliplatin (XELOX), which was subsequently amended to a $2 \times 2$ factorial design with further randomisation to bevacizumab or placebo. ${ }^{11}$

The aforementioned analysis by Loupakis et al. ${ }^{8}$ which included data from AVF2107g and NO16966, evaluated the association between tumour location and survival in $\mathrm{mCRC}$. Thus, all evaluable patients from AVF2107g and both part 1 (FOLOX4 vs XELOX) and part $2(2 \times 2$ factorial plus bevacizumab) of NO16966 were included. However, since our objective was to compare the effect

\footnotetext{
'Istituto Oncologico Veneto (IRCCS), Via Gattamelata, 64, 35128 Padova, PD, Italy; ${ }^{2}$ Duke University Medical Center, 10 Duke Medicine Circle, Durham, NC 27710, USA; ${ }^{3}$ Memorial Sloan Kettering Cancer Center, 1275 York Avenue, New York, NY 10021, USA; ${ }^{4}$ Asklepios Tumorzentrum Hamburg, AK Altona, Paul-Ehrlich-Str. 1, 22763 Hamburg, Germany; ${ }^{5}$ Mayo Clinic, 200 1st St SW, Rochester, MN 55902, USA; ${ }^{6}$ F. Hoffmann-La Roche, Ltd., Grenzacherstrasse 124, CH-4070 Basel, Switzerland and ${ }^{7}$ University of Southern California, Norris Comprehensive Cancer Center, Keck School of Medicine, 1441 Eastlake Ave, Los Angeles, CA 90033, USA

Correspondence: H.-J. Lenz (lenz@usc.edu)

${ }^{8}$ Present address: Genentech, Inc., 1 DNA Way, South San Francisco, CA 94080, USA
}

Received: 26 January 2018 Revised: 28 August 2018 Accepted: 24 September 2018

Published online: 29 November 2018 
of bevacizumab $+C T$ vs $C T$ in right- and left-sided tumours, our analysis focuses on patients who were concurrently randomised to bevacizumab $+\mathrm{CT}$. Patients who received CT only (FOLFOX4 or XELOX) from part 1 of NO16966 are excluded here.

Sidedness of patients from N016966 (NCT00069095) and AVF2107g (NCT00109070) was identified by Clinical Study Report information, including surgery procedural reports, updated from Loupakis et al. $^{8}$ Cancers proximal (i.e. occurring in the caecum, ascending colon, or transverse colon) or distal (i.e., occurring in the descending colon, sigmoid colon, or rectum) to the splenic flexure were defined as right- or left-sided, respectively. Tumours occurring precisely at the splenic flexure were assigned to the left-sided group. Patients with synchronous right- and left-sided tumours were excluded. Median progression-free survival (PFS) and OS, as well as corresponding $95 \% \mathrm{Cls}$, were estimated using Kaplan-Meier methods. Interaction tests were performed to determine whether treatment outcomes with bevacizumab + CT vs CT were different for patients with right- vs left-sided mCRC tumours. Unadjusted Cox proportional hazards models with right/ left terms were used to compare survival outcomes between treatment groups and were correlated with efficacy endpoints; corresponding $\mathrm{HRs}$ and $95 \% \mathrm{Cls}$ were estimated. In an overall treatment comparison for all patients with right- or left-sided tumours, tumour location was included as a covariate in the Cox model and interaction of treatment and tumour location was assessed. Overall response rate (ORR) was summarised as categorical data and compared between treatment groups by odds ratio (OR).

\section{RESULTS}

Sidedness was determined in 1590 patients (27\% right and $73 \%$ left) of 2214 patients total (Table 1). PFS was superior in patients treated with bevacizumb + CT vs CT in both right- (median PFS, 8.7 vs 5.8 months; $\mathrm{HR}=0.75 ; 95 \% \mathrm{Cl} 0.61,0.93 ; p=0.008$ ) and leftsided (median PFS, 10.0 vs 8.2 months; $\mathrm{HR}=0.76 ; 95 \% \mathrm{Cl} 0.67$, $0.86 ; p<0.001) \mathrm{mCRC}$ in this pooled analysis of N016966 and AVF2107g (Fig. 1a, b). OS was also numerically improved with bevacizumab $+C T$ vs $C T$ in right-sided $\mathrm{mCRC}$ (median OS, 18.3 vs 15.6 months; $\mathrm{HR}=0.82 ; 95 \% \mathrm{Cl} 0.65,1.03 ; p=0.085)$ and statistically significantly improved in left-sided mCRC (median OS, 23.5 vs 20.8 months; $\mathrm{HR}=0.85 ; 95 \% \mathrm{Cl} 0.74,0.98 ; p=0.028$ ) (Fig. 1c, d). The magnitude of PFS and OS benefit did not differ by tumour location.

To determine survival outcomes by baseline characteristics, patients were stratified by sex, age, race, Eastern Cooperative Oncology Group performance status, presence of colon cancer, prior adjuvant $\mathrm{CT}$, and prior radiation therapy. Compared with $\mathrm{CT}$, bevacizumab $+C T$ was associated with greater PFS and OS benefits for both right- and left-sided MCRC for most of these categories (Fig. 2).

In an overall treatment comparison for all patients with right- or left-sided tumours, tumour location was included as a covariate in the Cox model and interaction of treatment and tumour location was non-significant (PFS, $p=0.898$; OS, $p=0.697$ ). Cox model analysis also showed a non-significant (PFS, $p=0.980$; OS, $p=$ 0.879 ) interaction of bevacizumab use and availability of sidedness information (right/left vs. not classified). Interaction tests for PFS $(p=0.904)$ and OS $(p=0.689)$ indicated that treatment effect did not differ between patients based on tumour location. ORRs were similar with bevacizumab + CT vs CT in both right-sided $(40.9 \%$ vs 42.4\%; OR, 0.94; 95\% Cl 0.64-1.38; $p=0.770)$ and left-sided $(54.4 \%$ vs $52.0 \%$; OR, $1.10 ; 95 \% \mathrm{Cl} 0.88-1.39 ; p=0.410) \mathrm{mCRC}$

\section{DISCUSSION}

Right-sided tumours have been widely associated with worse prognosis compared with left-sided tumours in mCRC. ${ }^{8,12-14}$
Table 1. Survival outcomes

\begin{tabular}{|c|c|c|c|c|}
\hline & \multicolumn{2}{|l|}{ PFS } & \multicolumn{2}{|l|}{ OS } \\
\hline & CT & $\mathrm{Bev}+\mathrm{CT}$ & $\mathrm{CT}$ & $\mathrm{Bev}+\mathrm{CT}$ \\
\hline \multicolumn{5}{|l|}{ Right side } \\
\hline \multicolumn{5}{|l|}{ NO16966 } \\
\hline$N$ & 107 & 117 & 107 & 117 \\
\hline $\begin{array}{l}\text { Median } \\
(95 \% \mathrm{Cl})\end{array}$ & $\begin{array}{l}7.6 \\
(5.9,9.9)\end{array}$ & $\begin{array}{l}8.6 \\
(7.6,10.2)\end{array}$ & $\begin{array}{l}17.7 \\
(14.7,21.0)\end{array}$ & $\begin{array}{l}20.1 \\
(17.5,23.5)\end{array}$ \\
\hline \multicolumn{5}{|l|}{ AVF2107 } \\
\hline$N$ & 103 & 103 & 103 & 103 \\
\hline $\begin{array}{l}\text { Median } \\
(95 \% \mathrm{Cl})\end{array}$ & $\begin{array}{l}5.4 \\
(4.4,5.8)\end{array}$ & $\begin{array}{l}8.7 \\
(8.1,10.6)\end{array}$ & $\begin{array}{l}13.6 \\
(10.6,16.7)\end{array}$ & $\begin{array}{l}15.9 \\
(12.7,19.6)\end{array}$ \\
\hline \multicolumn{5}{|l|}{ Pooled } \\
\hline$N$ & 210 & 220 & 210 & 220 \\
\hline $\begin{array}{l}\text { Median } \\
(95 \% \mathrm{Cl})\end{array}$ & $\begin{array}{l}5.8 \\
(5.4,7.4)\end{array}$ & $\begin{array}{l}8.7 \\
(8.2,10.1)\end{array}$ & $\begin{array}{l}15.6 \\
(13.6,17.9)\end{array}$ & $\begin{array}{l}18.3 \\
(16.0,20.6)\end{array}$ \\
\hline $\begin{array}{l}\mathrm{HR}(95 \% \mathrm{Cl}) \\
p \text { value }\end{array}$ & \multicolumn{2}{|c|}{$\begin{array}{l}0.75 \\
(0.61,0.93) 0.008\end{array}$} & \multicolumn{2}{|c|}{$0.82(0.65,1.03) 0.085$} \\
\hline \multicolumn{5}{|l|}{ Left side } \\
\hline \multicolumn{5}{|l|}{ NO16966 } \\
\hline$N$ & 386 & 380 & 386 & 380 \\
\hline $\begin{array}{l}\text { Median } \\
(95 \% \mathrm{Cl})\end{array}$ & $\begin{array}{l}8.5 \\
(8.0,9.0)\end{array}$ & $\begin{array}{l}9.6 \\
(9.2,10.2)\end{array}$ & $\begin{array}{l}22.4 \\
(20.5,24.6)\end{array}$ & $\begin{array}{l}23.3 \\
(21.2,24.8)\end{array}$ \\
\hline \multicolumn{5}{|l|}{ AVF2107 } \\
\hline$N$ & 199 & 195 & 199 & 195 \\
\hline $\begin{array}{l}\text { Median } \\
(95 \% \mathrm{Cl})\end{array}$ & $\begin{array}{l}7.8 \\
(5.7,8.2)\end{array}$ & $\begin{array}{l}11.0 \\
(10.2,13.0)\end{array}$ & $\begin{array}{l}16.4 \\
(15.3,19.6)\end{array}$ & $\begin{array}{l}24.2 \\
(19.9, N R)\end{array}$ \\
\hline \multicolumn{5}{|l|}{ Pooled } \\
\hline$N$ & 585 & 575 & 585 & 575 \\
\hline $\begin{array}{l}\text { Median } \\
(95 \% \mathrm{Cl})\end{array}$ & $\begin{array}{l}8.2 \\
(7.9,8.5)\end{array}$ & $\begin{array}{l}10.0 \\
(9.4,10.8)\end{array}$ & $\begin{array}{l}20.8 \\
(19.6,22.4)\end{array}$ & $\begin{array}{l}23.5 \\
(21.6,24.8)\end{array}$ \\
\hline $\begin{array}{l}\mathrm{HR}(95 \% \mathrm{Cl}) \\
p \text { value }\end{array}$ & \multicolumn{2}{|c|}{$\begin{array}{l}0.76 \\
(0.67,0.86)<0.001\end{array}$} & \multicolumn{2}{|c|}{$\begin{array}{l}0.85 \\
(0.74,0.98) 0.028\end{array}$} \\
\hline
\end{tabular}

Bev bevacizumab, $\mathrm{Cl}$ confidence interval, $C T$ chemotherapy, $H R$ hazard ratio, PFS progression-free survival, OS overall survival

Meta-analysis showed that left-sided CRC was associated with a $20 \%$ reduction in the risk of death vs right-sided CRC, independent of disease stage, ethnicity, and study type. ${ }^{12}$ In our retrospective subgroup analysis of two randomised phase III mCRC studies, bevacizumab $+C T$ improved survival vs $C T$ alone, and the effect of bevacizumab was independent of tumour location. Similar improvements in PFS and OS were observed when patients were stratified according to baseline characteristics, such as age or race. In addition, OS was numerically improved with bevacizumab + CT vs $C T$ in right-sided mCRC (median OS, 18.3 vs 15.6 months; $p=$ 0.085 ) and significantly improved in left-sided mCRC (median OS, 23.5 vs 20.8 months; $p=0.028$ ). However, it is likely that the smaller magnitude of the right-sided $\mathrm{MCRC}$ patient subset contributed to the non-significant $p$ value for OS; therefore, these data suggest survival benefit overall and for both right- and leftsided tumours.

This is the first analysis of two large phase III mCRC studies comparing bevacizumab + CT vs CT alone by tumour location. As such, it represents a more comprehensive analysis of the effect of bevacizumab by tumour location than previous reports, which have been mixed. ${ }^{15-17}$ For instance, a retrospective analysis of mCRC patients treated with XELOX with or without bevacizumab suggested that bevacizumab may primarily benefit patients with left-sided primary tumours. ${ }^{16}$ However, in another study, PFS was 
a
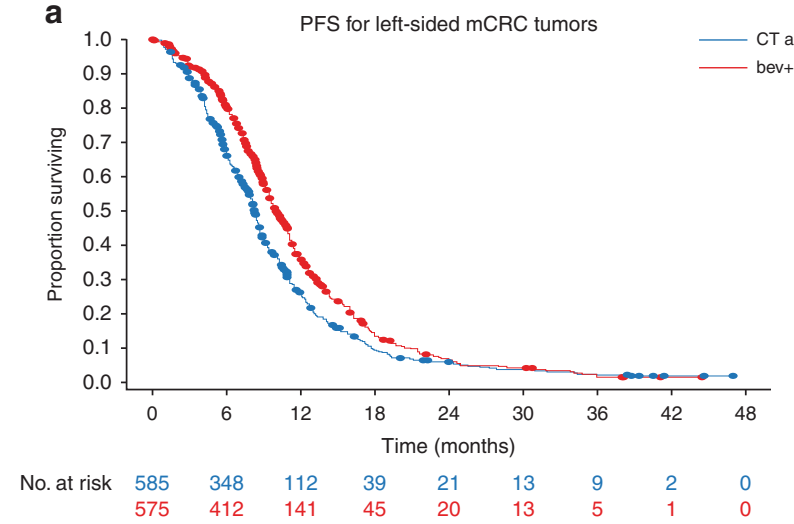

C

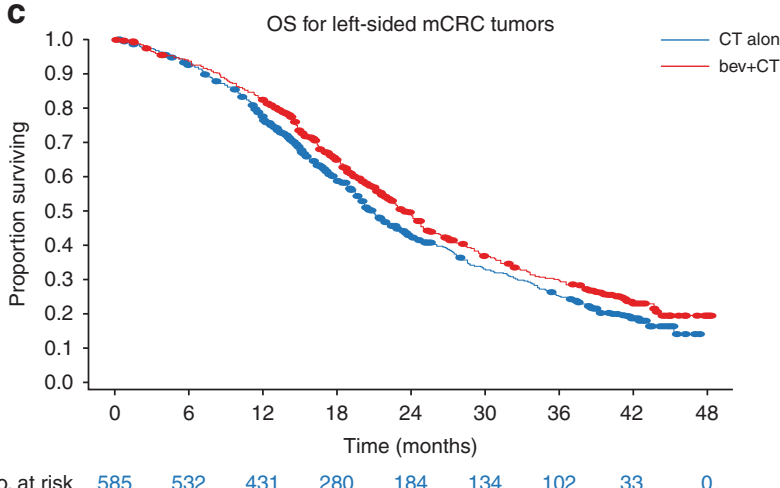

b

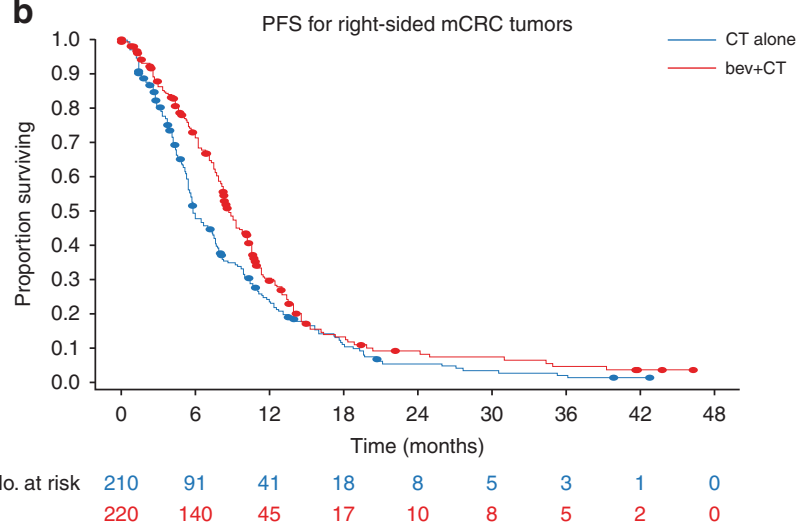

d

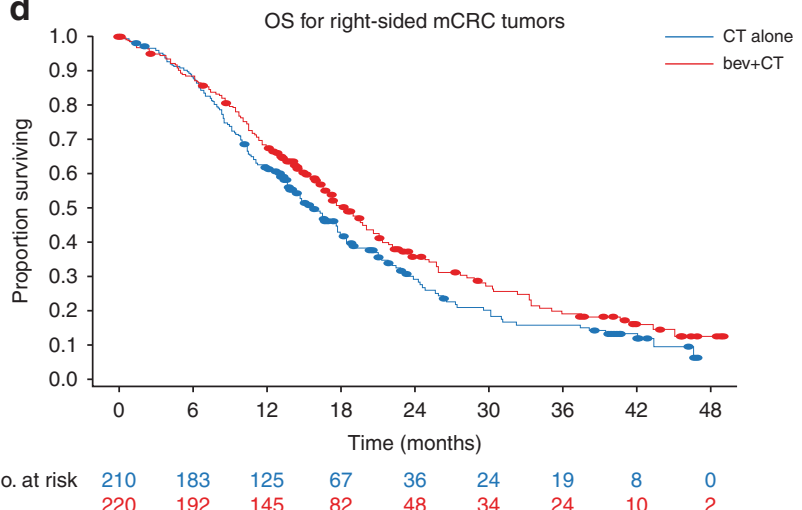

Fig. 1 Kaplan-Meier PFS and OS curves by tumour side for pooled population. a PFS for left-sided. $\mathbf{b}$ PFS for right-sided tumours. c OS for left-sided. d OS for right-sided. Bev bevacizumab, CT chemotherapy, OS overall survival, PFS progression-free survival

superior with bevacizumab $+\mathrm{CT}$ vs $\mathrm{CT}$ regardless of tumour site $(\mathrm{HR}=0.46){ }^{15}$ Our results build upon findings from the study by Loupakis et al. ${ }^{8}$ which showed that primary tumour location has a strong prognostic effect on patients with $\mathrm{mCRC}$ irrespective of bevacizumab exposure.

This study was a retrospective subgroup analysis, and was therefore limited to the patient populations investigated in the AVF2017g and N016966 trials. ${ }^{10,11}$ The tumour location of all patients was not identifiable; however, interaction tests showed no evidence of a relationship between the ability to identify the side and outcomes (PFS interaction, $p=0.980$; OS interaction, $p=$ 0.879). Although both AVF2107g and N016966 investigated bevacizumab, the CT backbones were different, which may have influenced the pooled results in our study. Another limitation is that data on RAS mutation status could not be included in our analysis. Since these data were not collected for NO16966, samples are not currently available for testing; for AVF2107g, sidedness information was only available for 180 out of 230 patients for whom KRAS data were collected, ${ }^{18}$ making the sample size too small for a valid analysis of the impact of treatment and RAS status. However, KRAS status has previously been shown not to be predictive for bevacizumab efficacy. ${ }^{19}$ Recent evidence points to the value of defining the sublocation of $\mathrm{mCRC}$ tumours, within the right- or left-sided classification method. In a study of 1876 CRC patients, the prevalence of mutations in BRAFV600, TP53, PIK3CA, and other genes differed by sublocation within right- and left-sided tumours. ${ }^{20}$ The authors concluded that the sigmoidrectal region of the left side appears unique, and the transverse colon is distinct from other right-sided locations. In our study, the lack of data on precise tumour location could be seen as a limitation. However, given that bevacizumab efficacy is maintained in both right- and left-sided tumours, further analyses by tumour sublocation would likely not influence the data presented here.

This retrospective exploratory subgroup analysis of two pivotal studies indicates the effect of bevacizumab is independent of tumour location in a non-biomarker selected mCRC population.

\section{ACKNOWLEDGEMENTS}

Linda Yau, PhD, of Genentech, Inc. contributed to the statistical analysis and provided critical feedback during early drafts of this manuscript. Medical writing assistance was provided by Sachi Yim of CodonMedical, an Ashfield Business, part of UDG Healthcare PLC, and supported by F Hoffmann-La Roche/Genentech.

\section{AUTHOR CONTRIBUTIONS}

All authors participated in the data collection, data analysis, and drafting of the manuscript. All authors also approved the final version of the manuscript to be submitted.

\section{ADDITIONAL INFORMATION}

Competing interests: F.L. served as a consultant/advisor for Roche, Amgen, and Bayer, and had travel, accommodations, and/or expenses paid for by Roche, Amgen, Merck Serono, and Bayer. H.I.H. is a current employee of Genentech/Roche and previously served on the advisory board of Genentech/Roche. L.S. received research funding from Taiho. D.A. received honoraria from, and served as a consultant or advisor for, BMS, Roche, Bayer, Merck, and EMD, and received research funding from BMS, Roche, Bayer, Merck, EMD, Incyte, and Taiho. Q.L.N., S.O., and J.T. are employees of Roche. S.S. is an employee of Roche and has a leadership role, owns stock, served as a consultant/advisor, received research funding, had travel/accommodations expenses paid, and had other relationships with Roche. H.-J.L. served on the advisory board for Bayer, Lilly, Merck, Roche, Sanofi, Servier, Sirtex, and Terumo; served as a lecturer for Bayer, Biocompatibles, Lilly, Merck, MSD, Roche, Sanofi, Servier, and Sirtex; 


\begin{tabular}{|c|c|c|c|c|}
\hline a & \multicolumn{3}{|c|}{ PFS for left-sided mCRC tumors } & \multirow[b]{2}{*}{$\mathrm{HR}(95 \% \mathrm{Cl})$} \\
\hline Category group & $\mathrm{n}$ & bev $+\mathrm{CT}$ & CT alone & \\
\hline \multicolumn{5}{|l|}{ Sex } \\
\hline Male & 725 & + & & $0.896(0.759-1.058)$ \\
\hline Female & 435 & + & & $0.718(0.580-0.888)$ \\
\hline \multicolumn{5}{|l|}{ Age, years } \\
\hline$\leq 50$ & 237 & + & & $0.651(0.486-0.871)$ \\
\hline $51-65$ & 573 & + & & $0.933(0.774-1.124)$ \\
\hline$>65$ & 350 & + & & $0.818(0.646-1.036)$ \\
\hline \multicolumn{5}{|r|}{ ) } \\
\hline White & 992 & + & & $0.858(0.745-0.987)$ \\
\hline Black & 46 & - & & $0.279(0.118-0.661)$ \\
\hline \multirow{2}{*}{\multicolumn{5}{|c|}{ ECOG PS }} \\
\hline & & & & \\
\hline 0 & 712 & + & & $0.883(0.746-1.046)$ \\
\hline$\geq 1$ & 446 & + & & $0.739(0.602-0.908)$ \\
\hline \multicolumn{5}{|l|}{ Colon cancer } \\
\hline \multirow{2}{*}{\multicolumn{5}{|c|}{ Prior adjuvant $\mathrm{CT}$}} \\
\hline & & & & \\
\hline Yes & 340 & + & & $1.011(0.791-1.294)$ \\
\hline \multirow{2}{*}{\multicolumn{5}{|c|}{ Prior radiation therapy }} \\
\hline & & & & \\
\hline Yes & 258 & + & & $1.184(0.883-1.588)$ \\
\hline No & 902 & + & & $0.767(0.663-0.888)$ \\
\hline \multirow[t]{2}{*}{ Overall } & 1160 & + & & $0.835(0.733-0.951)$ \\
\hline & & 0.1 & 10 & \\
\hline & & $\mathrm{HR}$ & & \\
\hline
\end{tabular}

b

\begin{tabular}{l} 
Category group \\
\hline Sex \\
Male \\
Female \\
Age, years \\
$\leq 50$ \\
$51-65$ \\
$>65$ \\
Race \\
White \\
Black \\
Other \\
ECOG PS \\
0 \\
$\geq 1$ \\
Colon cancer \\
Yes \\
Prior adjuvant CT \\
Yes \\
No \\
Prior radiation therapy \\
Yes \\
No \\
Overall
\end{tabular}

PFS for right-sided mCRC tumors

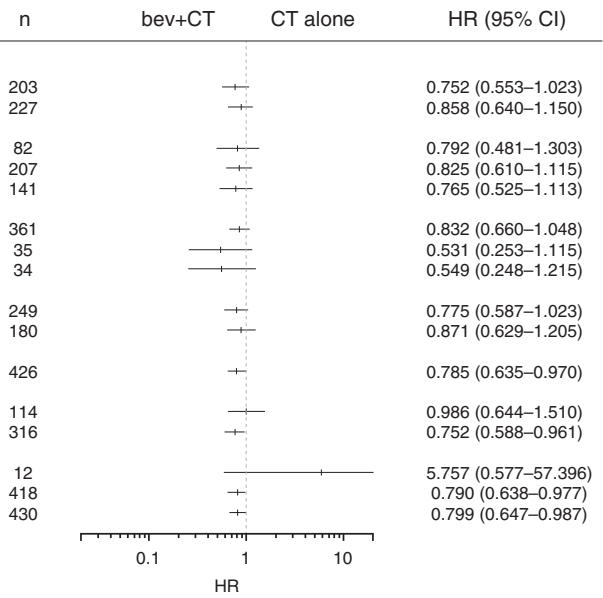

d

\begin{tabular}{l} 
Category group \\
\hline Sex \\
Male \\
Female \\
Age, years \\
$\leq 50$ \\
$51-65$ \\
$>65$ \\
Race \\
White \\
Black \\
Other \\
ECOG PS \\
0 \\
D1 \\
Colon cancer \\
Yes \\
Prior adjuvant CT \\
Yes \\
No \\
Prior radiation therapy \\
Yes \\
No \\
Overall \\
\end{tabular}

OS for right-sided mCRC tumors

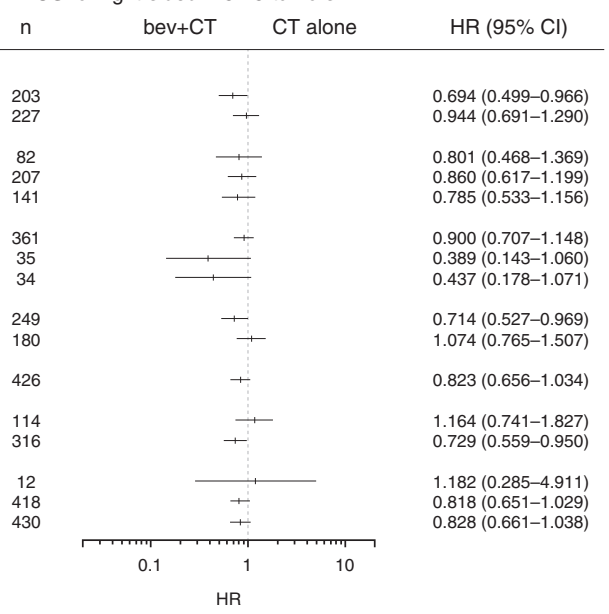

Fig. 2 Forest plots for PFS and OS by tumour side for pooled population. a PFS for left-sided. b PFS for right-sided. c OS for left-sided. (d) OS for right-sided. Bev, bevacizumab; CT, chemotherapy; OS, overall survival; PFS, progression-free survival

and received research funding from Mologen, Roche, and Sanofi. A.G. declares no competing interests.

Ethics approval: This study was conducted in accordance with International Conference on Harmonisation Good Clinical Practice regulations and the Declaration of Helsinki. The informed consent document and protocol were reviewed and approved by the Independent Ethics Committees of each study centre. All patients provided written informed consent before enrolment.

Data availability: Qualified researchers may request access to individual patient level data through the clinical study data request platform (www. clinicalstudydatarequest.com). Further details on Roche's criteria for eligible studies are available here (https://clinicalstudydatarequest.com/Study-Sponsors/StudySponsors-Roche.aspx). For further details on Roche's Global Policy on the Sharing of Clinical Information and how to request access to related clinical study documents, see here (https://www.roche.com/research_and_development/who_we_are_how_ we_work/clinical_trials/our_commitment_to_data_sharing.htm).

Funding: This study was supported by $\mathrm{F}$ Hoffmann-La Roche/Genentech. LS was funded in part by P30-17 CA008748.

\section{REFERENCES}

1. Arnold, D. et al. Prognostic and predictive value of primary tumour side in patients with RAS wild-type metastatic colorectal cancer treated with chemotherapy and EGFR directed antibodies in six randomised trials. Ann. Oncol. 28, 1713-1729 (2017).

2. Meza, R., Jeon, J., Renehan, A. G. \& Luebeck, G. Colorectal cancer incidence trends in the United States and United Kingdom: evidence of right- to left-sided biological gradients with implications for screening. Cancer Res. 70, 5419-5429 (2010).

3. Missiaglia, E. et al. Distal and proximal colon cancers differ in terms of molecular, pathological, and clinical features. Ann. Oncol. 25, 1995-2001 (2014).

4. Benedix, F. et al. Comparison of 17,641 patients with right- and left-sided colon cancer: differences in epidemiology, perioperative course, histology, and survival. Dis. Colon Rectum 53, 57-64 (2010).

5. Meguid, R. A., Slidell, M. B., Wolfgang, C. L., Chang, D. C. \& Ahuja, N. Is there a difference in survival between right- versus left-sided colon cancers? Ann. Surg. Oncol. 15, 2388-2394 (2008).

6. Dejea, C. M. et al. Microbiota organization is a distinct feature of proximal colorectal cancers. Proc. Natl Acad. Sci. USA 111, 18321-18326 (2014).

7. Mik, M., Berut, M., Dziki, L., Trzcinski, R. \& Dziki, A. Right- and left-sided colon cancer-clinical and pathological differences of the disease entity in one organ. Arch. Med. Sci. 1, 157-162 (2017).

8. Loupakis, F. et al. Primary tumor location as a prognostic factor in metastatic colorectal cancer. J. Natl Cancer Inst. 107, 1-9 (2015).

9. Tejpar, S. et al. Prognostic and predictive relevance of primary tumor location in patients with RAS wild-type metastatic colorectal cancer: retrospective analyses of the CRYSTAL and FIRE-3 trials. JAMA Oncol. 3, 194-201 (2017).

10. Hurwitz, $H$. et al. Bevacizumab plus irinotecan, fluorouracil, and leucovorin for metastatic colorectal cancer. N. Engl. J. Med. 350, 2335-2342 (2004). 
11. Saltz, L. B. et al. Bevacizumab in combination with oxaliplatin-based chemotherapy as first-line therapy in metastatic colorectal cancer; a randomized phase III study. J. Clin. Oncol. 26, 2013-2019 (2008).

12. Petrelli, F. et al. Prognostic survival associated with left-sided vs right-sided colon cancer: a systematic review and meta-analysis. JAMA Oncol. 3, 211-219 (2017).

13. Modest, D. P. et al. Outcome of patients with metastatic colorectal cance depends on the primary tumor site (midgut vs. hindgut): analysis of the FIRE1trial (FuFIRI or mIROX as first-line treatment). Anticancer Drugs 25, 212-218 (2014).

14. Yahagi, M., Okabayashi, K., Hasegawa, H., Tsuruta, M. \& Kitagawa, Y. The worse prognosis of right-sided compared with left-sided colon cancers: a systematic review and meta-analysis. J. Gastrointest. Surg. 20, 648-655 (2016).

15. Wong, H. L. et al. Impact of primary tumor site on bevacizumab efficacy in metastatic colorectal cancer. Clin. Colorectal Cancer 15, e9-e15 (2016).

16. Boisen, M. K. et al. Primary tumor location and bevacizumab effectiveness in patients with metastatic colorectal cancer. Ann. Oncol. 24, 2554-2559 (2013).

17. Holch, J. W., In, Ricard, Stintzing, S., Modest, D. P. \& Heinemann, V. The relevance of primary tumour location in patients with metastatic colorectal cancer: a metaanalysis of first-line clinical trials. Eur. J. Cancer 70, 87-98 (2017).

18. Hurwitz, H. I., Yi, J., Ince, W., Novotny, W. F. \& Rosen, O. The clinical benefit of bevacizumb in metastatic colorectal cancer is independent of K-ras mutation status: analysis of a phase III study of bevacizumab with chemotherapy in previously untreated metastatic colorectal cancer. Oncologist 14, 22-28 (2009).
19. Hurwitz, H. I. et al. Efficacy and safety of bevacizumab in metastatic colorectal cancer: pooled analysis from seven randomized controlled trials. Oncologist 18, 1004-1012 (2013).

20. Loree, J. M. et al. Classifying colorectal cancer by tumor location rather than sidedness highlights a continuum in mutation profiles and Consensus Molecular Subtypes. Clin. Cancer Res. 24, 1062-1072 (2018).

Open Access This article is licensed under a Creative Commons Attribution 4.0 International License, which permits use, sharing, adaptation, distribution and reproduction in any medium or format, as long as you give appropriate credit to the original author(s) and the source, provide a link to the Creative Commons license, and indicate if changes were made. The images or other third party material in this article are included in the article's Creative Commons license, unless indicated otherwise in a credit line to the material. If material is not included in the article's Creative Commons license and your intended use is not permitted by statutory regulation or exceeds the permitted use, you will need to obtain permission directly from the copyright holder. To view a copy of this license, visit http://creativecommons. org/licenses/by/4.0/.

(c) The Author(s) 2018 